Case Report

\title{
Post-caesarean Section Cicatricial Parietal Endometriosis: Case Report and Review of the Literature at the Brazzaville University Hospital (Republic of Congo)
}

\author{
Buambo Gauthier Régis Jostin ${ }^{1, ~ *, ~ E o u a n i ~ M a x ~ L e ́ v y ~ E m e ́ r y ², ~ M o u a m b a ~ F a b i e n ~ G a e ̈ l ~}{ }^{3}$, \\ Ali-Zaoro Fayçal Khali1 ${ }^{1}$, Mokoko Jules César ${ }^{1}$, Itoua Clautaire ${ }^{1}$, Pecko Jean Félix ${ }^{3}$, \\ Iloki Léon Hervé ${ }^{1}$ \\ ${ }^{1}$ Department of Gynecology Obstetrics, University Hospital of Brazzaville, Brazzaville, Congo \\ ${ }^{2}$ Department of Gynecology Obstetrics, Loandjili Hospital, Pointe Noire, Congo \\ ${ }^{3}$ Department of Pathological Cytology and Anatomy, University Hospital of Brazzaville, Brazzaville, Congo
}

\section{Email address:}

buambogauthier@yahoo.fr (Buambo G. R. J.), eouani@yahoo.fr (Eouani M. L. E.), mfabiengael@gmail.com (Mouamba F. G.), mmaina244@gmail.com (Ali-Zaoro F. K.),jlsmokoko@gmail.com (Mokoko J. C.), clautairei@yahoo.com (Itoua C.), jfpecko@hotmail.fr (Pecko J. F.), herviloki@yahoo.fr (Iloki L. H.)

${ }^{*}$ Corresponding author

\section{To cite this article:}

Buambo Gauthier Régis Jostin, Eouani Max Lévy Eméry, Mouamba Fabien Gaël, Ali-Zaoro Fayçal Khalil, Mokoko Jules César, Itoua Clautaire, Pecko Jean Félix, Iloki Léon Hervé. Post-caesarean Section Cicatricial Parietal Endometriosis: Case Report and Review of the Literature at the Brazzaville University Hospital (Republic of Congo). Journal of Gynecology and Obstetrics. Vol. 8, No. 1, 2020 , pp. $24-27$. doi: $10.11648 /$ j.jgo.20200801.16

Received: January 17, 2020; Accepted: February 7, 2020; Published: February 14, 2020

\begin{abstract}
Introduction: Defined as the implantation of endometrial tissue within parietal abdominal structures more superficial than the peritoneum, parietal endometriosis is an exceptional localization of extragenital endometriosis, most often secondary to gynecological obstetric surgery. Clinical observation: We report the case of a 32-year-old patient presenting with an abdominal parietal pain syndrome in comparison with a post-caesarean cutaneous cicatrix, cyclic and rhythmic by menstruation. Ultrasonography of the soft tissues revealed two anterior, heterogeneous hypoechoic nodules with posterior reinforcement. A wide surgery with $1 \mathrm{~cm}$ of healthy margin allowed the excision of the nodule interesting the subcutaneous tissue and the aponeurosis of the rectus abdominis muscle. The histology was in favour of a focal endometriosis of the abdominal wall extended to the fascia of the rectus muscle. Microscopic examination revealed endometrial glands of variable size, sometimes dilated, bordered by regular cylindrical epithelium, associated with a cytogenic chorion and lymphocytic inflammation, sometimes with blood. Immediate postoperative outcomes were simple. An LHRH analogue protocol (Leuprolide acetate $3.75 \mathrm{mg}$ in one intramuscular injection every 28 days for 6 months) was instituted. Conclusion: Although exceptional, it is appropriate to think of an endometriosis before an abdominal pain syndrome abdominal wall of the woman during periods of genital activity.
\end{abstract}

Keywords: Endometriosis, Abdominal Wall, Scar, Caesarean Section, Brazzaville

\section{Introduction}

Endometriosis is defined as the presence of ectopic endometrial tissue located distant from the endometrium and without connection with it [1]. Its most frequent location is in the internal genitalia. It affects the ovaries, the peritoneum, the uterine ligaments, the rectovaginal lamina. Other extra-genital locations are less frequent. They have been described, in the bladder, intestine, appendix, navel, hernial bags, lung, kidneys and abdominal wall [2]. Parietal involvement represents 1 to $2 \%$ of extra-genital locations [3]. Its occurrence in gynecological or obstetrical surgery scars is rare, ranging from 0.03 to $0.4 \%[4,5]$. Therefore, it is considered iatrogenic, involving the transport and grafting of endometrial fragments 
[6]. We report the case of a patient with endometriosis of the abdominal wall on Pfannenstiel post-caesarean scar. This case is reported because of its rarity and the unusual nature of its location.

\section{Clinical Observation}

This is a 32-year-old patient, fourth gesture and second par, with a history of two miscarriages caused by aspiration. She has two live children born by caesarean section with unspecified indications, the last of which was three years old.

For six months after the last caesarean section, the patient

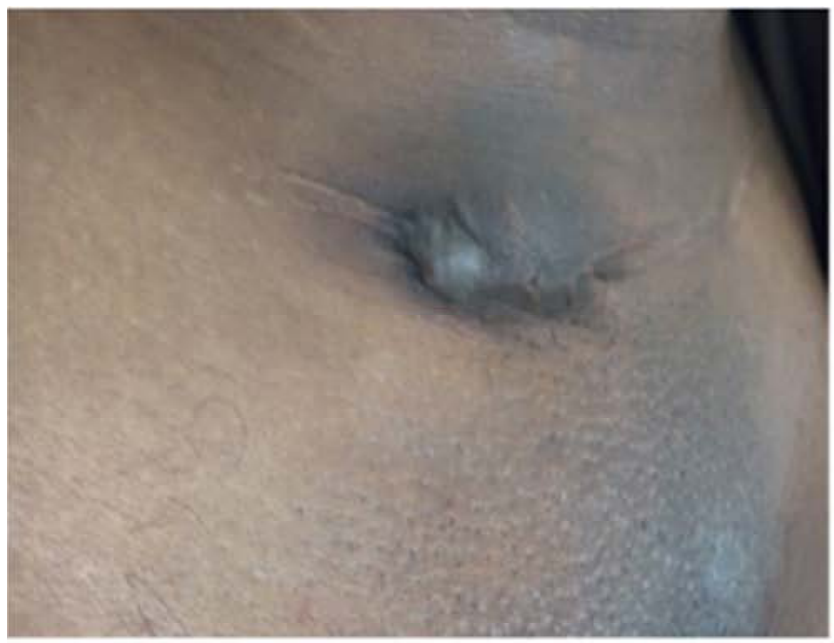

A complains of pain in the cutaneous incision scar, cyclic and rhythmic with menstruation, with a variable intensity of up to 6 on the analogue verbal scale (EVA), intermittent, cramp-like, localized with progressive swelling of the contemporary scar of pain. She did not report deep dyspareunia, pain with bowel movement, or dysuria.

The clinical examination revealed a mass opposite the right end of the cutaneous scar about $3 \mathrm{~cm}$ long, fixed in the deep plane. In the menstrual period, the mass increases in volume up to $5 \mathrm{~cm}$ long axis, inflammatory appearance, renitent consistency, sensitive (Figure 1).

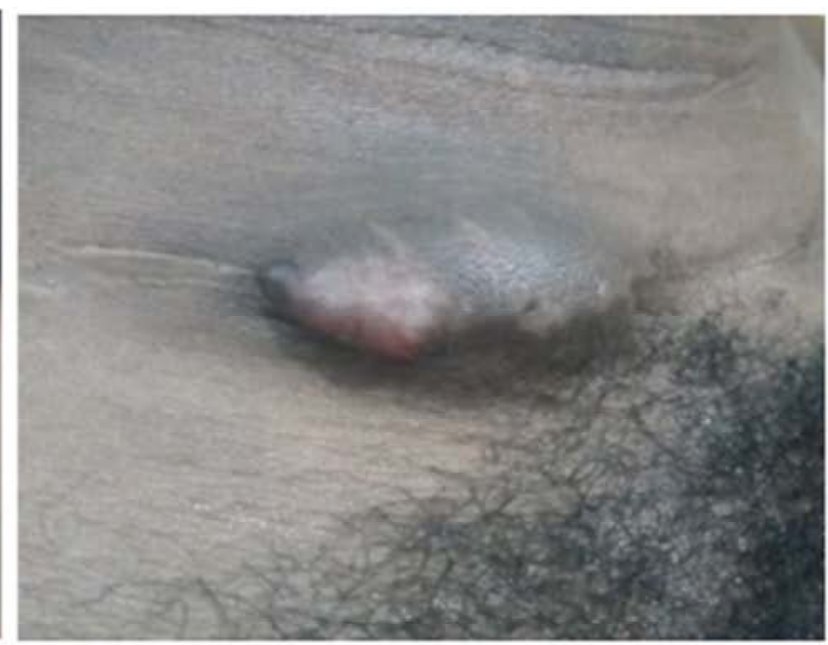

B

Figure 1. Post-caesarean section cicatricial parietal endometriosis.

A. Before menstruation.

B. After menstruation.

Ultrasonography of the soft tissues revealed two anterior, heterogeneous hypoechoic nodules with posterior reinforcement measuring: $15.9 \mathrm{~mm} \times 25.2 \mathrm{~mm}$ and $18.6 \mathrm{~mm} \times 33.7 \mathrm{~mm}$ (Figure 2).

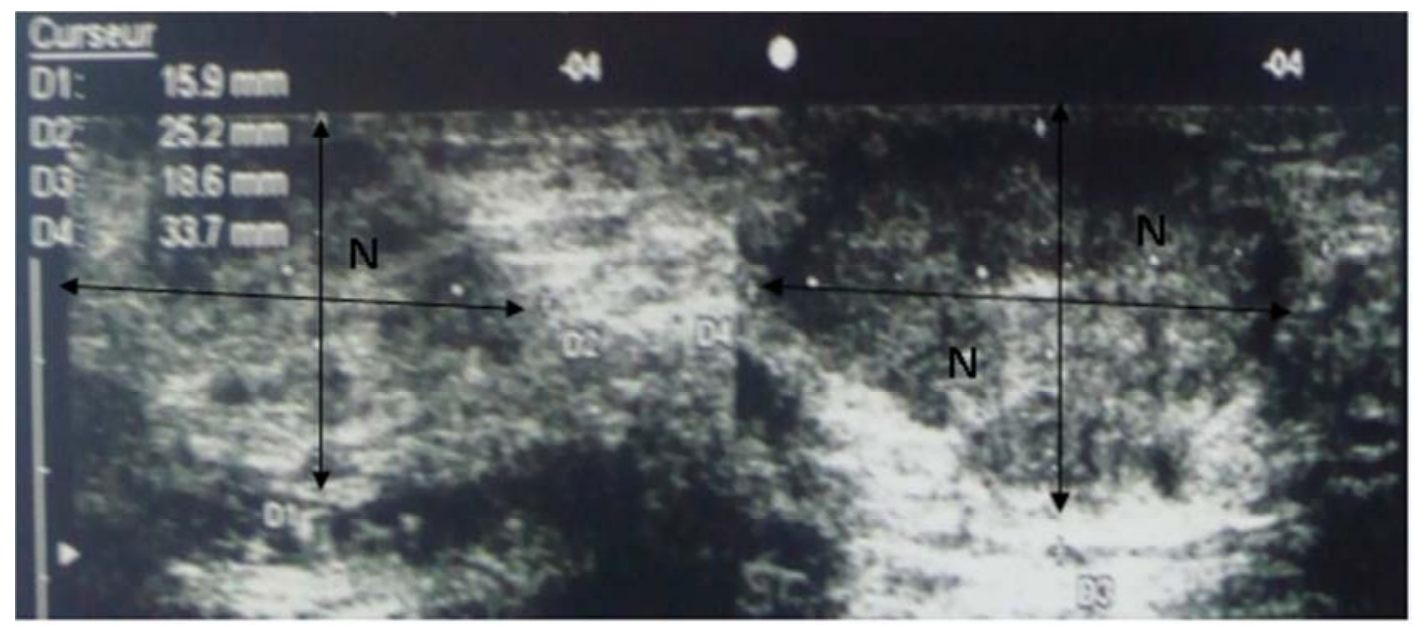

Figure 2. Soft tissue ultrasound. N: Endometriosis Nodule.

The decision of a surgical resection was made. It was a bilobed nodule, interesting subcutaneous tissue and aponeurosis of the right muscle, without muscle involvement. A wide excision respecting $1 \mathrm{~cm}$ of healthy zone in depth and taking away part of the muscle was carried out (Figure 3). 


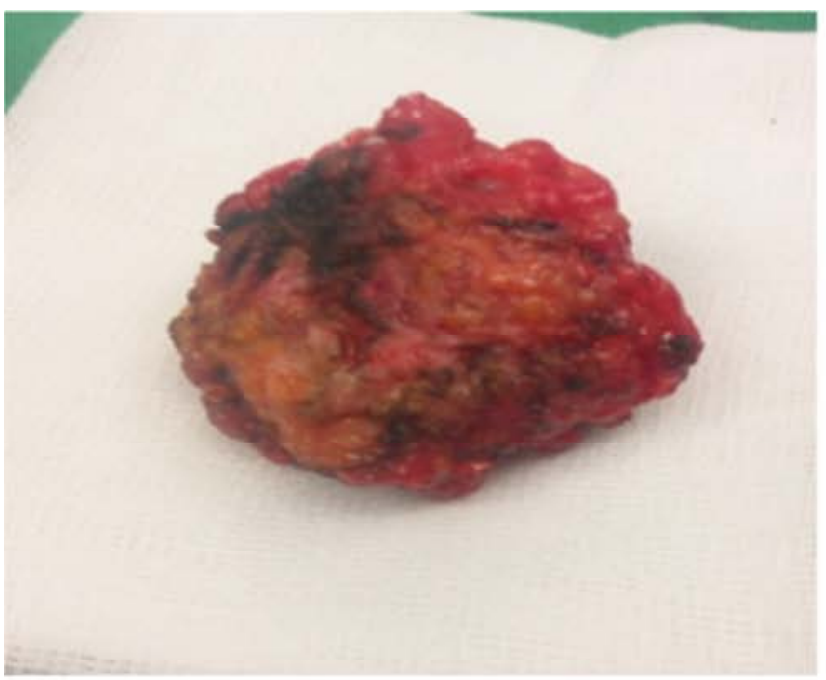

A

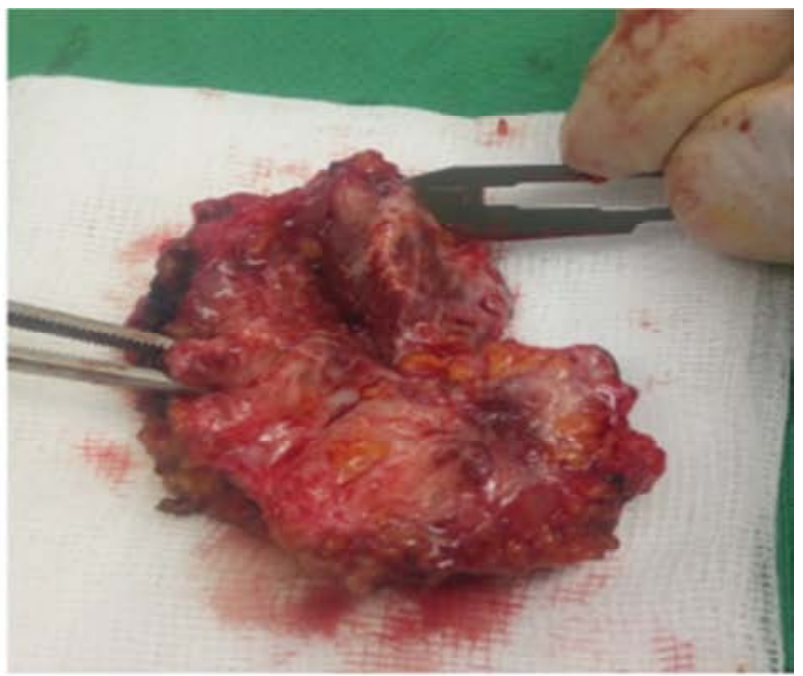

B

Figure 3. Surgical excision piece.

A. Before dissection.

B. After dissection.

The histology was in favour of a focal endometriosis of the abdominal wall (Figure 4) extended to the fascia of the rectus muscle. Immediate postoperative outcomes were simple. An LHRH analogue protocol (Leuprolide acetate $3.75 \mathrm{mg}$ in one intramuscular injection every 28 days for 6 months) was instituted. A control at three months and then every six months was performed, with no recurrence of a decline of 16 months.

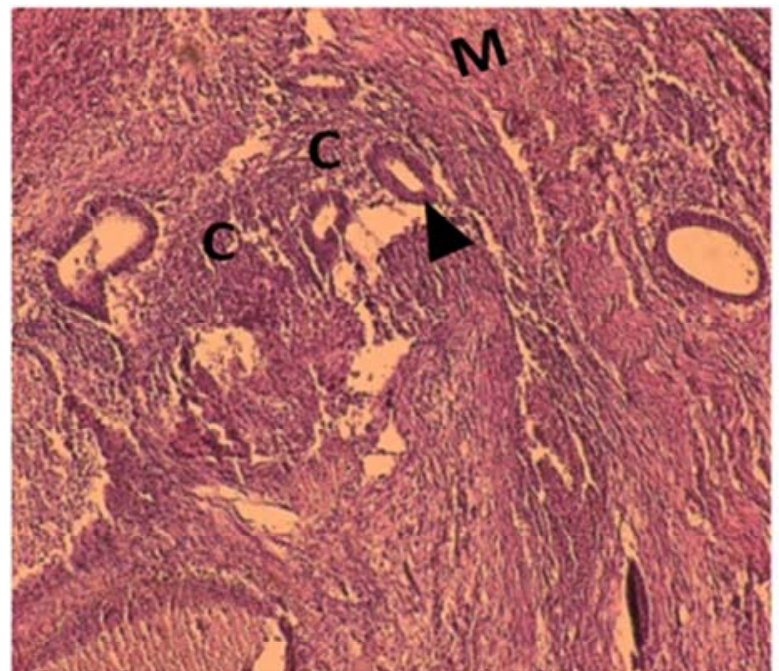

A

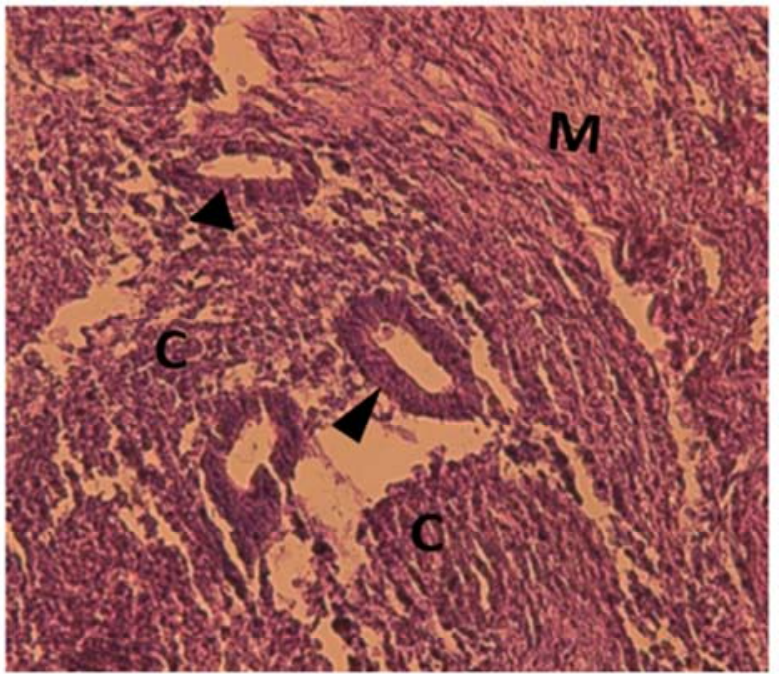

B

Figure 4. Histological sections.

A. $(\operatorname{HEx} 100)$.

B. $($ HEx 250$)$.

Arrowhead: Endometrial glands. C: Cytogenic chorion. M: Abdominal parietal mesenchymal tissue.

\section{Discussion}

Defined as an ectopic implantation of endometrial tissue outside the uterine cavity, endometriosis affects approximately 2 to $15 \%$ of women in genital activity $[1,6,7]$. Several extragenital locations have been described: pelvic peritoneum, bladder, rectum, nothing, gallbladder, lung, stomach and brain [8]. The parietal endometriosis, rare, includes the incisional attacks (consecutive to a gynaeco-obstetrical surgery), largely majority, and the spontaneous endometriomas. Nearly $70 \%$ of cases of abdominal wall endometriosis occur on operative scars, 
according to a review of 455 cases published from 1951 to 2006, of which 57\% after caesarean section [9].

As a result, cicatricial parietal involvement is induced and iatrogenic. This induction theory is based on local grafting of endometrial cells that will develop in non-epithelialized zones [3]. Their development is also favoured by secondary inflammation induced by immunological factors. Also, retrograde menstruation of endometrial cells by tubal reflux, tubal plated against the peritoneum; explain this location $[8$, 10]. The existence of the metaplastic (mesenchymal cell differentiation under the influence of hormonal factors) and metastatic (embolization by the lymphatic and venous embolization) theories were also evoked in the case of umbilical endometriotic nodules and right-sided muscle sheaths., in the absence of surgery [8].

Regarding the clinic, the usual circumstances of the diagnosis are unambiguous and associate pathognomonic catamenial pain symptomatology with cyclic fluctuation of the volume of the mass or nodule located at the level of the scar. These signs appear several months to several years after the imputed surgical procedure [11].

The diagnosis is based on imaging and histopathological examination after surgical excision of the mass. Colour Doppler ultrasound appears as the examination of choice, showing a hypoechoic mass. Magnetic resonance imaging (MRI) is more informative, with sensitivity and specificity of $90 \%$ and $98 \%$, respectively [12], found a T1 hypersignal in favour of intralesional bleeding and also makes it possible to specify the depth extension of the 'endometrioma.

Pathologically, the endometrioma macroscopically presents itself as a pseudo cystic tumour, of fibrous appearance at the cut, containing congestive or necrotic-haemorrhagic foci. All parietal structures can be affected (muscles, fascia, fascia, subcutaneous tissues). Microscopic examination reveals endometrial glands of variable size, sometimes dilated, lined by regular cylindrical epithelium, associated with a cytogenic chorion and lymphocytic inflammation, sometimes with blood, confirming the diagnosis.

Medical treatments (antigonadotrophic, LH-RH analogy) of parietal locations have shown their limit, exposing patients to frequent recurrence, side effects of hormone-suppression [13] or androgenic effects. Medical treatment can be conceived as an induction treatment for bulky masses, aimed at reducing the volume of the endometrioma and the parietal defect resulting from its removal. In addition, the exceptional endometrioid carcinoma, reported for extragenital localization [14], constitutes an additional argument in favour of surgical excision. The resection should be broad from the outset, "carcinologic" taking away muscle and fascia to prevent recurrence.

\section{Conclusion}

Parietal endometriosis is exceptional, and its mechanisms of occurrence are well understood. Although the diagnosis is histological, the occurrence of an abdominal parietal pain syndrome in a woman during a period of genital activity, should evoke a parietal endometriosis and perform ultrasound or magnetic resonance imaging, for excision wide surgical.

\section{Conflicts of Interest}

All the authors do not have any possible conflicts of interest.

\section{References}

[1] Paillocher N, Paris L, Bouission F, Cotici V, Croué A, Anglade E, Catala L and Descamps P. Endométriomes Ovariens. EMC (Elsevier Masson SAS, Paris), Gynécologie, 150-A-40, 2006.

[2] Lamblin G, Mathevet P, Buenerd A. Parietal Endometriosis on Abdominal Scar, About Three Observations. J Gynecol Obstet Biol Reprod 1999; 28: 271-4.

[3] Mounia Z, Abdellah B, Mehdi H, Jaouad K, Driss RM, Mohamed D. Parietal Endometriosis about Seven Cases and Review of the Literature. Global Journal of Medical Research (E) Gynecology and Obstetrics 2016; 16 (1): 32-8.

[4] El Fahssi M, Lomdo M, Bounaim A, Ali AA and Sair K. Parietal - Scar Endometriosis After Cesarean Section: A Rare Entity. Pan Afr Med J 2016; 24: 79.

[5] Jayi S, Laadioui M, Bouguern H, Chaara H and Melhouf A. Endometriosis of the Abdominal Wall: About A Rare Case. Pan Afr Med J 2013; 15: 86.

[6] Audebert A. Iatrogenic Endometriosis During Reproductive Age: Main Issues? Gynecol Obstet Fertil 2013; 41 (5): 322-7.

[7] Hafidi R, Kouach J, Yakka M, Salek G, Oukabli M, Moussaoui R-D, Dehayni M. Endometriosis of an Abdominal Wall Scar. Imagerie de la Femme (2011) 21, 125-7.

[8] Durand X, Daligand H, Aubert P, Baranger B. Abdominal Wall Endometriosis. J Visc Surg 2010; 147: 354-9.

[9] Horton JD, Dezee KJ, Ahnfeldt EP, Wagner M. Abdominal Wall Endometriosis: A Surgeon's Perspective and Review of 455 Cases. Am J Surg 2008; 1 (96): 207-12.

[10] Patterson GK, Winburn GB. Abdominal Wall Endometriomas: Report of Eight Cases. Am Surg 1999; 65: 36-9.

[11] Bouhout T, Serji B, Egyir EU, El Amri B, Bouhout I, Soufi M, Bouziane $M$ and El Harroudi $M$. Abdominal Wall Endometriosis: about a case. Pan Afr Med J 2018; 30: 4.

[12] Onbas O, Kantarci M, Alper F, Kumtepe Y, Durur I, Ingec M, and al. Nodular Endometriosis: Dynamic MR Imaging. Abdom imaging 2007; 32 (4): 451-6.

[13] Witz CA. Current Concepts in the Pathogenesis of Endometriosis. Clin Obstet Gynecol 1999; 42 (3): 566-85.

[14] Kotwall CA, Kirkbride P, Zerafa AE, Murray D. Endometrial Cancer and Abdominal Wound Recurrence. Gynecol Oncol 1994; 53 (3): 357-60. 